\title{
¿Afectan las medidas reparatorias no pecuniarias ordenadas en un arbitraje de inversión a la soberanía de los Estados? Perspectivas desde la experiencia ecuatoriana
}

\author{
Andrés Larrea Savinovich* \\ Juan Martín Alarcón Peralta** \\ Recibido/Received: 30/06/2019 \\ Aceptado/Accepted: 01/07/2019
}

\begin{abstract}
SumARIO: 1. Introducción. 2. Medidas reparatorias. 2.1 Medidas reparatorias pecuniarias. 2.2 Medidas reparatorias no pecuniarias en arbitraje de inversión. 2.2.1 Ejecutabilidad de medidas reparatorias no económicas. 3. ¿Qué significa la soberanía estatal? 4. Preocupación sobre injerencia en la soberanía. 5. Análisis casuístico de los casos de arbitraje donde ha participado el Ecuador. 5.1 Caso Perenco ARB 08/06.5.2 Caso City Oriente ARB 06/21.5.3 Caso Occidental Petroleum ARB 06/11. 6. Conclusión.
\end{abstract}

Resumen: El presente artículo analiza las medidas reparatorias no pecuniarias en el arbitraje internacional de inversiones vis à vis la soberanía de los Estados, haciendo énfasis en casos en los que el Ecuador ha sido parte. Las preguntas a analizar son: ¿Atentan las medidas no pecuniarias dictadas

* Asociado Senior en Tobar ZVS Spingarn. Profesor titular de Introducción al Derecho de la Facultad de Derecho de la Universidad Técnica Particular de Loja. Secretario arbitral de la Cámara de Comercio de Quito. Abogado por la Universidad de las Américas, LL.M., por McGill University. Correo electrónico: alarrea@tzvs.ec

** Asociado en Tobar ZVS Spingarn. Abogado por la Universidad San Francisco de Quito. Correo electrónico: juanmartinap13@gmail.com

A. Larrea Savinovich \& J. M. Alarcón Peralta, “¿Afectan las medidas reparatorias no pecuniarias ordenadas en un arbitraje de inversión a la soberanía de los Estados? Perspectivas desde la experiencia ecuatoriana", Revista Ecuatoriana de Arbitraje, No. 10, 2019, pp. 213-232. 
por tribunales de arbitraje de inversión a la soberanía estatal? ¿Cuál es la experiencia del Ecuador en relación a las medidas no pecuniarias en arbitraje de inversión? Para responder estas preguntas, en primer lugar, se analizará la naturaleza de las distintas medidas de reparación en el arbitraje internacional de inversiones. En segundo lugar, se hará un análisis casuístico de los procesos arbitrales en los cuales el Ecuador ha sido parte, para explorar la aplicabilidad de este tipo de reparaciones. Finalmente, se estudiará el concepto de soberanía dentro del ordenamiento jurídico nacional e internacional. Con este análisis, se demostrará que las medidas de reparación no pecuniarias son una alternativa viable en muchos casos y además, constituyen un mecanismo idóneo para preservar la relación entre el Estado y el inversionista.

Palabras Clave: Ecuador, arbitraje de inversiones, medidas reparatorias no pecuniarias, daños, soberanía estatal.

\section{Do non-pecuniary remedies ordered in an investment arbitration affect State sovereignty? Perspectives from the Ecuadorean experience}

Aвstract: This article analyzes the nature of non-pecuniary remedies in international investment arbitration vis-á-vis the idea of sovereignty, with an emphasis on cases where Ecuador has been a party. Our research questions are: Do non-pecuniary remedies in international investment arbitration threaten the States' sovereignty? What is the Ecuadorian experience with respect to non-pecuniary remedies in investment arbitration? In order to answer these questions, we will analyze the different types of remedies available in international investment arbitration. Then, we will conduct an empirical research on investment arbitrations cases where the Ecuadorian State has been a party, in order to explore the applicability of these types of remedies. Finally, we will analyze the concept of sovereignty 
within the international and domestic legal framework in order to conclude that non-pecuniary remedies are available and, in many cases, these remedies are the most appropriate mechanism to preserve the investor-state relationship.

KEYWORDS: Ecuador, investment arbitration, non-pecuniary remedies, torts, State sovereignty.

\section{INTRODUCCIÓN}

La soberanía de los Estados es un concepto dispar y con varias interpretaciones en el mundo jurídico internacional. No existe una definición uniforme respecto al significado de esta palabra. Sin embargo, existen algunos elementos en común que la mayoría de países reconocen al momento de definir el significado de soberanía. Así, se encuentra que para la mayoría de países la soberanía se entiende como aquella capacidad o "poder" que tienen los Estados de tomar decisiones de última instancia, sin sometimiento a consulta ${ }^{1}$. En el Ecuador, parecería que la soberanía se afinca en la voluntad de sus mandantes, el pueblo. Tan laxo como suena el concepto de soberanía, esta palabra ha servido como un justificativo para que muchos Estados se opongan a cuanta medida, obligación y orden que sea dictada por terceros contraria a sus intereses.

El fin último en materia de daños, es alcanzar el restitutio in integrum ${ }^{2}$, el cual no se utiliza de manera inequívoca en el derecho internacional, toda vez que tiene dos acepciones: (i) tiene que ver con la restitución plena, es decir, retomar el estado inmediatamente anterior a la irrigación del daño; y, (ii) tiene que ver con la restitución en

1. V. Naranjo, Teoría Constitucional e Instituciones Políticas, Editorial Temis, 1995, p. 210.

2. R. Águlla, Los límites al principio de reparación integral, Santiago, 2010, p. 28. 
especie que es sinónimo del cumplimiento específico ${ }^{3}$. Sin embargo, parecería que los tribunales arbitrales no toman en cuenta que, en muchos casos, las medidas reparatorias no pecuniarias son las más eficaces para lograr este objetivo. Esto se evidencia de la gran parte de los laudos dictados por el Centro Internacional de Arreglo de Diferencias Relativas a Inversiones (en adelante, CIADI), en los cuales rara vez se ordenan este tipo de medidas. La principal razón para este fenómeno es la discusión que ha surgido sobre la posible vulneración a la soberanía estatal mediante la aplicación de medidas de reparación no pecuniarias.

El convenio CIADI no ha previsto disposición alguna que faculte o impida a los tribunales arbitrales para aplicar medidas resarcitorias no económicas. Con lo cual, se concluye que les está permitido a los tribunales arbitrales a ordenarlas. Por otro lado, el Tratado de Libre Comercio de América del Norte (en adelante, TLCAN) ha excluido expresamente la restitución jurídica ${ }^{4}$, monetizando los daños en el cien por ciento de los casos; y, por tanto, ha cerrado la puerta a la aplicación de medidas reparatorias no pecuniarias. Por ello, la problemática jurídica tratada en el presente artículo es relevante, pues no existe armonía ni uniformidad en la legislación internacional aplicable.

Esta investigación no pretende concluir que las medidas reparatorias deben ser aplicadas obligatoriamente en todos los casos para alcanzar la restitución integral. Al contrario, lo que busca demostrar es que estas medidas forman parte del catálogo de opciones que tienen los árbitros a disposición y que, su adopción debe ser considerada en base a su eficacia y no a un temor infundado de menoscabar la soberanía estatal.

3. Occidental Petroleum Corporation and Occidental Exploration and Production Company v. Ecuador, Caso CIADI No. ARB 06/1, Decisión sobre medidas provisionales, 17/08/2007, párr. 77-89.

4. Tratado de Libre Comercio de América del Norte (1992). Art. 1135. 


\section{Medidas Reparatorias}

Las medidas reparatorias son la vía mediante la cual un tribunal o un juez puede hacer cumplir un derecho ante la perpetración de un daño o lesión, que haya sido reconocido como consecuencia de un ilícito. El objetivo principal es compensar o restituir al afectado. Según el diccionario jurídico Black's Law Dictionary, restitución es la "acción de restaurar; devolución de algo a su legítimo dueño; acción de hacer el bieno dar el equivalente por pérdida, daño o injuria;indemnización" ${ }^{\prime \prime}$. Para lograr dicho fin, se puede optar por diferentes opciones, como la orden de compensar económicamente al afectado o restituirlo a su estado previo a la irrigación del daño, esto es lo que se conoce en derecho como restitución jurídica ${ }^{6}$.

\subsection{Medidas reparatorias pecuniarias}

Cuando se ha irrigado un daño al patrimonio, lo más común, es que se ordene la reparación del detrimento mediante medidas pecuniarias o de carácter económico, es decir, este tipo de reparaciones implican una obligación de dar (dinero) por parte del deudor. El fin de estas medidas es compensar las afectaciones patrimoniales de quien ha sufrido un daño. En la legislación ecuatoriana, el artículo 336 del Código Orgánico Administrativo prevé que en caso de no poder restituir las cosas a su estado original se procederá al pago de una indemnización ${ }^{7}$. La debida cuantificación del importe a pagar es una tarea meramente discrecional del juez o tribunal's. Si bien se deberá hacer una correcta subsunción de los hechos, el derecho y el daño perpetuado, la cuantificación de la compensación económica siempre quedará al buen criterio del juzgador.

5. Traducción libre. B. Garner \& H. CAmpBell, Black's Law Dictionary, 1999.

6. S. Napolitano, Responsabilidad internacional del Estado por actos ilícitos, Universidad de Buenos Aires, 2011 p, 103.

7. Código Orgánico Administrativo, Art. 336, RO No. 31, 07/07/2017.

8. Revista de Derecho y Jurisprudencia, Tomo 95, No. 1, p. 38. 
Para evitar que el criterio del tercero imparcial radique en la arbitrariedad, se ha contrastado este método mediante la implementación de baremos, mismos que pretenden tazar los posibles daños con anterioridad`. En el mismo sentido, la publicidad de las sentencias resarcitorias es otro mecanismo de homologar criterios a la hora de ordenar una medida resarcitoria pecuniaria ${ }^{10}$. Sin embargo, ninguno de los métodos descritos logra materializar un sistema inequívoco de tasación. Motivo por el cual, no siempre las medidas de reparación económicas son el camino más eficaz para reparar y compensar el daño.

Es un hecho notorio que las medidas de reparación pecuniarias son las más adoptadas por los juzgadores. Sin embargo, no son ellos los únicos responsables, pues frente a la ocurrencia de un daño, lo más común es que las partes y sus abogados soliciten en sus demandas, compensaciones de carácter económico. Por ello, los juzgadores se encuentran limitados a pronunciarse exclusivamente sobre la pretensión de la demanda, so pena de caer en vicios de incongruencia al momento de decidir como el extra petita.

\subsection{Medidas reparatorias no pecuniarias en arbitraje de inversión}

En primer lugar, es menester indicar que, en el derecho internacional, cuando se ha producido un daño, nace la obligación de reparación por parte de quien lo ha provocado. Para entender esta obligación, es importante referirnos brevemente al fallo de la Corte Permanente de Justicia Internacional (en adelante, CPJI) también conocido como Chorzow, probablemente uno de los primeros referentes en

9. H. CoRral TAlCian, Lecciones de responsabilidad civil extracontractual, Editorial Jurídica de Chile, 2004.

10. Ibídem. 
esta materia ${ }^{11}$. En la sentencia, la CPJI señala el principio de reparación como una obligación en derecho internacional:

[E]s un principio de derecho internacional, e incluso una concepción general de derecho, que toda violación de un compromiso implica obligación de reparar en forma adecuada; [...] la reparación debe, en la medida de lo posible, borrar todas las consecuencias del acto ilícito y restablecer la situación que, según toda probabilidad, habría existido si dicho acto no se hubiera cometido $[\ldots]^{12}$.

Por lo tanto, en derecho internacional el fin último de la reparación integral es restituir al afectado, volviendo las cosas al estado anterior al cometimiento del daño ${ }^{13}$. Si bien en muchos casos esa reparación comprende una compensación de carácter económico, en otros casos es necesario adoptar una serie de medidas no pecuniarias para extinguir las consecuencias de algún acto ilegal. Estas medidas de reparación no materiales juegan un papel importante y son, en muchos casos, las más apropiadas para alcanzar la restitución. Por un lado, la restitución será de carácter material cuando esté relacionada, por ejemplo, con una propiedad confiscada por un Estado o la liberación de una persona encarcelada ${ }^{14}$. Por otro lado, la restitución será de carácter jurídico cuando busque restablecer el status quo existente previo al daño ${ }^{15}$.

Para entender el concepto de restitución integral, es importante mencionar que existen distintos tipos de medidas no pecuniarias ${ }^{16}$. Por ejemplo, el cese de una actividad o el cumplimiento específico. En relación al cese, un tribunal

11. C. NAsh, Las Reparaciones ante la Corte Interamericana de Derechos Humanos, 2da Ed., Universidad de Chile, Centro de Derechos Humanos, 2009.

12. Tribunal Permanente de Justicia Internacional, Alemania v. Polonia, 13/09/1928, párr. 68, <https://bit.ly/2E24ORR> (17/07/2019).

13. Z. Becerra, "La Obligación de Reparar como Principio del Derecho Internacional", Revista Jurídica de la Universidad de León, No. 3, 2016.

14. T. Ishikawa, "Restitution as a Second Chance for Investor-State Relations: Restitution and Monetary Damages as Sequential Options", McGill Journal of Dispute Resolution, Vol. 3, 20162017.

15. Ibídem.

16. T. IsHiKaWA, N. 14. 
de arbitraje puede ordenar que un Estado se abstenga de realizar una determinada conducta ${ }^{17}$. El cese implica que un Estado detenga la conducta que está provocando el daño. Para los expertos en la materia, el cese no está enfocado a las consecuencias de la conducta dañosa y es más bien una reparación de carácter puramente legal ${ }^{18}$. En relación al cumplimiento específico, este es otra forma de reparación mediante el cual se busca proteger los derechos de un inversionista que han sido menoscabados por una decisión o acto estatal. Por lo tanto, lo que pretende este mecanismo es volver las cosas al estado anterior a la violación del derecho. Este tipo de restitución requiere de una intervención estatal mediante actos legislativos o administrativos para que se pueda restaurar la situación al momento anterior ${ }^{19}$. La restitución jurídica implica por lo tanto una modificación de una situación jurídica.

Como hemos anticipado, el convenio CIADI, así como otros acuerdos o tratados bilaterales de inversiones (en adelante, TBIs), no establecen ninguna prohibición para que los árbitros dicten medidas de naturaleza distinta a las que condenan al pago de una suma determinada de dinero. Por lo tanto, al no existir prohibición expresa en la mayoría de tratados internacionales, los tribunales tienen todas las facultades, como jueces privados que son, para ordenar las medidas que consideren necesarias de reparación ${ }^{20}$. Tomando en cuenta, además, que en el derecho internacional se ha reconocido que la reparación podrá comprender la adopción de medidas de carácter no pecuniario para restablecer el status $q u o^{21}$.

17. S. Willis-ValderRama, "Protección a la Inversión Extranjera en Infraestructura por Medio de Acuerdos Internacionales de Inversión: un Nuevo Reto para Colombia", Revista Colombiana de Derecho Internacional, No. 19, 2011.

18. B. AlLen, The Use of Non-pecuniary Remedies in WTO Dispute Settlement: Lessons for Arbitral Practitioners, $<$ https://bit.ly/2Snltno $>(01 / 05 / 2011)$.

19. Ibídem.

20. M. Blackaby \& A. Camargo, "Alternativas de Reparación en el Arbitraje Internacional de Inversiones", $A C D I$, No. 1, 2008.

21. Ibídem. 
En este sentido, en el caso Burlington contra Ecuador ${ }^{22}$ (ARB 08/5), el tribunal realiza un test de procedencia para conceder medidas no pecuniarias. Para el efecto, se deben cumplir tres requisitos esenciales:

(i) Existencia de un derecho a proteger

a. El derecho de exclusividad conforme el artículo 26 del CIADI;

b. El derecho a que no se agrave la disputa y a mantener el status quo; $\mathrm{y}$,

c. El derecho al cumplimiento específico y a la efectividad del laudo.

(ii) Urgencia por preservar el derecho

(iii) Test de necesidad y eficacia

\subsubsection{Ejecutabilidad de las medidas reparatorias no económicas}

Existe controversia en relación a la ejecutabailidad de este tipo de medidas. Después de todo, de nada sirve que los árbitros opten por ordenar reparaciones no pecuniarias si en la práctica se tornan inejecutables por cualquier razón que fuere ${ }^{23}$. En principio, son ejecutables estas órdenes en la medida en la que sean posibles. Sería inútil considerar una medida no pecuniaria cuando no es materialmente posible cumplirla, por ejemplo, ordenar que se restituya una propiedad que se encuentra destruida o en posesión de un tercero ${ }^{24}$. De igual manera, no sirve de nada ordenar una medida de este tipo si la misma va a implicar un perjuicio mayor en proporción a una compensación

22. Burlington Resources Inc. v. República del Ecuador y Empresa Estatal de Petróleos Petroecuador, Caso CIADI ARB 08/5, Resolución Procesal No. 1 sobre medidas provisionales, 29/06/2009.

23. T. ISHIKAWA, N. 14.

24. J. RuEDA, "La Aplicabilidad del Convenio de Nueva York al Arbitraje de Inversiones: Efectos de las Reservas al Convenio", Cuadernos de Derecho Transnacional, Vol. 2, No. 1, 2010. 
económica ${ }^{25}$. Además, siempre está la preocupación por parte de algunos tribunales de incurrir en el vicio de ultra petita por ordenar algo distinto a lo reclamado ${ }^{26}$. En concordancia, la Comisión de Derecho Internacional de las Naciones Unidas, en el artículo 35 del Tratado de Responsabilidad del Estado por Hechos Internacionalmente Ilícitos prescribe:

El Estado responsable de un hecho internacionalmente ilícito está obligado a la restitución, es decir, a restablecer la situación que existía antes de la comisión del hecho ilícito, siempre que y en la medida en que esa restitución:

a. No sea materialmente imposible;

b. No entrañe una carga totalmente desproporcionada con relación al beneficio que derivaría de la restitución en vez de la indemnización ${ }^{27}$.

\section{El art. 51 numeral 1 del convenio CIADI señala que}

Todo Estado Contratante reconocerá al laudo dictado conforme a este Convenio con carácter obligatorio y hará ejecutar dentro de sus territorios las obligaciones pecuniarias impuestas por el laudo como si se tratare de una sentencia dictada por un tribunal existente en dicho Estado ${ }^{28}$.

A simple vista, parece que sólo las obligaciones de carácter monetario podrán ser ejecutadas por los Estados, dificultando así la adopción de medidas de carácter distinto. No obstante, hacer una interpretación tan restrictiva en relación a este artículo, iría en contra del sentido del propio convenio debido a que existen casos en los cuales la medida no pecuniaria es de imposible cumplimiento por lo que una obligación legal en de estas características carecería de sentido ${ }^{29}$. Sin embargo, en

\footnotetext{
25. H. Corral Talciani, N. 9.

26. Ibídem.

27. Informe de Responsabilidad del Estado por Hechos Internacionalmente Ilícitos de la Comisión de Derecho Internacional de las Naciones Unidas (2001), Art. 35.

28. Convenio sobre Arreglo de Diferencias Relativas a inversiones entre Estados y Nacionales de otros Estados (1966), Art. 51(1).

29. H. Corral Talciani, N. 9.
} 
el caso Libia v. Texaco ${ }^{30}$, el tribunal arbitral consideró que, para alcanzar la restitución integral, lo que procedía es la imposición al gobierno de Libia, de obligaciones de cumplimiento específico. Lo mismo sucedió en el caso Goetz v. Burundi ${ }^{31}$, en el cual el Tribunal optó por medidas no pecuniarias de forma subsidiaria a las compensaciones económicas.

\section{3. ¿QUÉ SIGNIFICA LA SOBERANÍA ESTATAL?}

El término soberanía proviene del latín super omina cuyo significado es: sobretodo o poder supremo. Jean Bodin ha definido a la soberanía de manera amplia como el "poder absoluto y perpetuo de una república" ${ }^{32}$. Para Bodin, soberano es el poder de decisión, el emitir leyes mas no recibirlas de otro ${ }^{33}$. Dos siglos después, Rousseau redefine al concepto de soberanía dándole un giro drástico. Para Rousseau, la soberanía radica en las personas y es la colectividad en su conjunto, la que ha decidido, por voluntad propia, enajenar sus derechos en favor de la autoridad. La Constitución de la República del Ecuador de 2008 recoge lo anunciado previamente en su primer artículo, el cual prescribe "la soberanía radica en su pueblo, cuya voluntad es el fundamento de la autoridad" ${ }^{34}$.

Ahora bien, en ejercicio de la soberanía de sus individuos, expide la carta magna, que prevé el deber que tiene el Estado de "garantizar y defender la soberanía nacional" 35 . Hasta aquí, no deja de ser un concepto abstracto que no lleva mayores

30. Texaco Overseas Petroleum Company \& California Asiatic Oil Company v. Gobierno de Libia, Tribunal Independiente, Laudo, 19/01/1977.

31. Antonio Goetz \& Otros v. República de Burundi, Caso CIADI No. ARB/95/03, Laudo, 10/02/1999.

32. J. Bodín, Los seis libros de la República, Madrid, Editorial Tecnos, 1992.

33. Ibídem.

34. Constitución de la República de Ecuador, Art. 1, RO No. 449, 10/20/2008.

35. Constitución de la República de Ecuador, Art. 3, RO No. 449, 10/20/2008.

Art. 3.- Son deberes primordiales del Estado: [...]

2. Garantizar y defender la soberanía nacional [...]. 
consecuencias prácticas; sin embargo, el artículo 422 de la Constitución de la República del Ecuador manda lo siguiente:

No se podrá celebrar tratados o instrumentos internacionales en los que el Estado ecuatoriano ceda jurisdicción soberana a instancias de arbitraje internacional, en controversias contractuales o de índole comercial, entre el Estado y personas naturales o jurídicas privadas ${ }^{36}$.

Este artículo fue redactado bajo una errónea concepción sobre el significado de la soberanía. Toda vez que, el someter los conflictos respecto de los inversionistas a tribunales extranjeros no compromete, en absoluto, la soberanía estatal ${ }^{37}$. Y menos aún dicha soberanía pende de la jurisdicción donde se lleve a cabo el proceso arbitral. Para GuASTINI, este concepto constitucionalista y el concepto de soberanía que se maneja en el derecho internacional, son completamente diferentes, pues tienen diferentes alcances y consecuencias ${ }^{38}$. Y es que la soberanía dentro del derecho constitucional tiene que ver con ese poder indelegable de crear normas, ese poder soberano que corresponde a uno solo. Ese poder de ser constituyente, no tener ningún fundamento de legitimidad precedente ni estar condicionado a la decisión de terceros.

Es relevante para el presente artículo, el concepto de soberanía en el marco del derecho internacional que nos atañe. El derecho internacional enfrenta una dicotomía: (i) Por un lado la teoría de KELSEN que establece que los Estados no son soberanos y, en consecuencia, se deben al derecho internacional. Toda vez que sus ordenamientos no son originarios en absoluto, se fundamentan en normas de derecho internacional. Siendo entonces el poder constitucional de los Estados, un poder constituido ${ }^{39}$. Y, por otro

36. Constitución de la República de Ecuador, Art. 422, RO No. 449, 10/20/2008.

37. O. E. García-Bolívar, "Sovereignty vs. Investment Protection: Back to Calvo?", ICSID Review-Foreign Investment Journal, Washington, D.C., 2009, p. 468.

38. R. Guastini, La sintaxis del derecho, Marcial Pons, 2016, p. 312.

39. H. Kelsen, Il problema della soveranità e la teoría del diritto internazionale, Milano, 1920. Traducción al español por H. CAminos \& E. C. Hermida,"Estudio Preliminar: Soberanía y De- 
lado, según FERRAJOLI, (ii) los Estados sí son soberanos y por tal, no obedecen a obligación proveniente del derecho internacional alguna siendo completamente libres ${ }^{40}$. Ninguno de estos conceptos resulta del todo convincente, en este sentido, GUASTINI determina que "la soberanía es el presupuesto -la condición sine qua nonpara la aplicabilidad de normas internacionales, incluidas aquellas que imponen obligaciones a los Estados"41.

Este concepto de soberanía que admite la imposición de obligaciones en el ámbito internacional -muy aparte de las obligaciones convencionales, claro está- calza perfectamente con la historia del Estado ecuatoriano. No será ajeno para muchos, la propuesta de un arbitraje papal entre el Ecuador y el Perú iniciado en 1991 ante la Asamblea General de la Organización de las Naciones Unidas, para presentar sus disputas limítrofes a consideración de un tercero imparcial: el Papa ${ }^{42}$. ¿Qué otro aspecto puede ser más intrínseco al concepto de soberanía que la limitación territorial de un Estado? Probablemente ninguno. Así, concluimos que cuando un Estado pacta un convenio arbitral y somete su disputa a la jurisdicción de un tribunal, no está renunciando a su soberanía sino todo lo contrario, lo hace en pleno ejercicio de la misma.

\section{Preocupación sobre injerencia en la soberanía}

Si bien hemos mencionado algunas razones (facilidad en la ejecución, términos del contrato o tratado, ley aplicable) por las cuales existe una tendencia por parte de los árbitros a no dictar medidas no pecuniarias en materia de arbitraje de

recho Internacional en Hans Kelsen: mito y realidad” a cargo de J. L. Monereo Pérez, Editorial Comares, 2013.

40. L. Ferrajoli, "La soberanía en el mundo moderno", Derechos y Garantías. La ley del más débil, 1era Ed., Trotta, 1999, pp. 125-175.

41. R. Guastini, N. 38, p. 316.

42. Véase, R. BorJA, Ecuador-Perú: El arbitraje papal, Afese, <https://bit.ly/2JUAyZY> $(18 / 07 / 2019)$. 
inversión, no podemos desconocer que una de dichas razones, es su preocupación por evitar una injerencia en la soberanía. Después de todo, ordenar que un Estado modifique, revoque o altere un acto administrativo o deje sin efecto alguna resolución administrativa o política pública, implica entrar en un análisis teórico y controversial respecto a si dicha orden constituye una intrusión en la soberanía.

Como hemos dicho, son raros los casos en los que los tribunales ordenan reparaciones no pecuniarias, sin embargo, la tendencia tradicional de sólo ordenar compensaciones económicas trae muchas desventajas. En ocasiones el daño sufrido por los inversionistas es difícil de cuantificar en dinero. Por ejemplo, si un Estado viola su obligación contenida en un tratado e impide que los extranjeros asuman posiciones gerenciales en la compañía de un inversionista, la cuantificación en dinero no es más apropiada que ordenar al Estado que deje sin efecto tal conducta ${ }^{43}$. Esto se debe a que las reparaciones económicas usualmente tratan las consecuencias del pasado y no evitan que cierta conducta dañosa se repita en el futuro. Por lo tanto, el temor de los tribunales a dictar estas medidas termina siendo perjudicial en muchos casos para ambas partes.

\section{Análisis casuístico de los casos de arbitraje donde HA PARTICIPADO EL ECUADOR}

El presente cuadro tabula la información recabada respecto de todos los procesos arbitrales seguidos bajo el convenio CIADI en los cuales ha participado el Ecuador:

43. Occidental v. Ecuador. N. 3.

*** Abreviaciones:

MP Medida Pecuniaria

MPNP Medida Provisional No Pecuniaria

DNC Declaratoria de No Competencia

SP Suspensión del Proceso

T Transacción 


\begin{tabular}{|c|c|c|c|}
\hline No. caso & Demandante & Reparación ${ }^{* * *}$ & Tipo \\
\hline ARB $/ 06 / 11$ & $\begin{array}{l}\text { Occidental } \\
\text { Petroleum } \\
\text { Corporation }\end{array}$ & MP & \\
\hline $\mathrm{ARB} / 09 / 23$ & $\begin{array}{l}\text { Corporación } \\
\text { Quiport S.A. }\end{array}$ & $\mathrm{T}$ & \\
\hline $\mathrm{ARB} / 08 / 10$ & $\begin{array}{l}\text { Repsol YPF } \\
\text { Ecuador, S.A. }\end{array}$ & $\mathrm{T}$ & \\
\hline $\mathrm{ARB} / 08 / 06$ & $\begin{array}{c}\text { Perenco Ecuador } \\
\text { Limited } \\
\end{array}$ & MPNP & \\
\hline $\mathrm{ARB} / 08 / 05$ & $\begin{array}{c}\text { Burlington } \\
\text { Resources, Inc. }\end{array}$ & MP & Cese \\
\hline $\mathrm{ARB} / 08 / 04$ & $\begin{array}{l}\text { Murphy } \\
\text { Exploration }\end{array}$ & $\mathrm{DNC}$ & \\
\hline $\mathrm{ARB} / 03 / 06$ & $\begin{array}{c}\text { M.C.I. Power } \\
\text { Group, L.C. and } \\
\text { New Turbine, Inc. }\end{array}$ & DVL & \\
\hline $\mathrm{ARB} / 06 / 21$ & $\begin{array}{l}\text { City Oriente } \\
\text { Limited }\end{array}$ & MPNP & $\begin{array}{c}\text { Cese } \\
\text { Cumplimiento } \\
\text { específico }\end{array}$ \\
\hline $\mathrm{ARB} / 02 / 10$ & IBM World Trade & $\mathrm{T}$ & \\
\hline $\mathrm{ARB} / 01 / 10$ & Repsol IPF & MP & \\
\hline $\mathrm{ARB} / 06 / 17$ & $\begin{array}{c}\text { Técnicas Reunidas } \\
\text { y Eurocontrol }\end{array}$ & SP & \\
\hline $\mathrm{ARB} / 05 / 12$ & $\begin{array}{l}\text { Noble Energy y } \\
\text { Machala Power }\end{array}$ & SP & \\
\hline $\mathrm{ARB} / 05 / 09$ & Emelec & $\mathrm{DNC}$ & \\
\hline ARB / 04/19 & $\begin{array}{c}\text { Duke Energy } \\
\text { Electroquil }\end{array}$ & MP & \\
\hline
\end{tabular}

Fuente: Base de datos del CIADI. Elaboración por los autores

De los casos antes referidos, se desprende que el Ecuador tiene catorce casos en el archivo de causas del $\mathrm{CIADI}^{44}$, en los

\footnotetext{
44. Centro Internacional de Arreglo de Diferencias Relativas a Inversionees, The ICSID Caseload - Statistics, Issue 2018-1, <https://bit.ly/2HS5k2y> (18/07/2019).
} 
cuales únicamente en dos los tribunales han ordenado como medida provisional resarcitoria el cese de una actividad por parte del Ecuador. Estas cifras representan apenas el 14\%. Si tomamos en cuenta los casos en los que el tribunal consideró las medidas no pecuniarias como una opción, pero no optó por ordenarlas, esta cifra asciende al 28\%; lo que quiere decir que en el resto de causas $(72 \%)$ no se han considerado si quiera la posibilidad de reparar al afectado a través de otro mecanismo que no sea la compensación económica. Al replicar esta tendencia de manera hipotética, nos lleva a concluir que de los 744 casos registrados en la base de datos del CIADI, apenas 104 casos posiblemente han considerado las medidas no pecuniarias como herramienta para la restitución. En consecuencia, estas cifras evidencian que, si bien los tribunales están facultados para ordenar medidas no pecuniarias, esto sucede en un porcentaje mínimo. Para entender el razonamiento de los tribunales en relación a la adopción de medidas reparatorias no pecuniarias, es menester analizar los siguientes casos.

\subsection{Caso Perenco ARB 08/06}

En el caso petrolero iniciado en el 2008 entre Perenco Ecuador Limited y la República del Ecuador, el tribunal arbitral dictó medidas resarcitorias no pecuniarias en contra del Ecuador con el objetivo de no agravar la contienda entre las partes ni vulnerar los derechos que los demandantes buscaban proteger mediante el arbitraje ${ }^{46}$. Las medidas ordenadas por el tribunal corresponden al cese de una actividad, toda vez que las mismas determinan prohibiciones específicas para las partes. Dichas medidas sin duda limitan el accionar del Estado, y no por esto se ha vulnerado la tan protegida soberanía ecuatoriana.

45. Perenco Ecuador Limited v. República del Ecuador, Caso CIADI No. ARB/08/06, Decisiones sobre las medidas provisionales, 8/05/2009.

46. Ídem, párr. 79 . 
Dentro del Caso Perenco, el Estado ecuatoriano alegó que las medidas solicitadas por parte de los demandantes no caben ya que la limitación a la aplicación de la Ley 42 interferiría con la independencia y soberanía del Estado, especialmente, con su poder legislativo; más aún, cuando la Corte Constitucional ecuatoriana determinó la vigencia y constitucionalidad de la Ley 42. Contrastando lo alegado por el Estado, el tribunal arbitral concluyó que si bien la Ley 42 goza de un pronunciamiento constitucional favorable, éste no puede limitar al tribunal arbitral de conceder medidas para mantener el status $q u o^{47}$. Existen precedentes internacionales donde los tribunales arbitrales han impedido que se aplique una ley promulgada en defensa de la soberanía estatal de promulgar leyes dentro de su territorio como: City Oriente v. Ecuador ${ }^{48}$ (versa sobre la misma Ley 42); y, Sergei Paushok y otros v. Mongolia ${ }^{49}$.

\subsection{Caso City Oriente ARB 06/21 $1^{50}$}

En el caso iniciado por City Oriente Limited v. República del Ecuador y la Empresa Estatal de Petróleos Petroecuador, el Tribunal optó por ordenar al Ecuador que se abstenga (cese) de iniciar cualquier acción judicial que involucre a la compañía inversionista y/o a sus directivos y/o a sus empleados y que guarde relación con el contrato materia del arbitraje ${ }^{51}$. A criterio del Tribunal, esta medida era necesaria para preservar el status quo, evitando así que se lleve a cabo cualquier acto que perjudique los derechos en disputa y que pueda agravar la controversia. Además, el Tribunal ordenó en este caso el cumplimiento específico para que las partes continúen con las

47. Ídem, párr. 25.

48. City Oriente Limited v. Ecuador, Caso CIADI No. ARB/06/21. Resolución sobre Medidas Provisionales, 19/11/2007.

49. Sergei Paushok, CJSC Golden East Company y CJSC Vostokneftegaz Company v. Gobierno de Mongolia, Caso CNUDMI, 28/04/2011.

50. City Oriente Limited v. Ecuador, N. 48.

51. Ídem, párr. 93. 
obligaciones asumidas en virtud del contrato. Por ejemplo, el tribunal ordenó que City Oriente Limited pague cualquier cantidad derivada de la aplicación al contrato de 29 de marzo de 1995 de la Ley No 2006-4252.

\subsection{Caso Occidental Petroleum ARB 06/11 ${ }^{53}$}

Es claro, que no todos los tribunales coinciden en que este tipo de medidas no afectan a la soberanía estatal. Por ejemplo, dentro del caso Occidental Petroleum Corporation y Occidental Exploration and Production Company v. República del Ecuador, el tribunal indicó que cuando un Estado, ejerciendo su soberanía, pone fin a un contrato, licencia o concesión, el "cumplimiento específico" es jurídicamente inviable ${ }^{54}$. Además, agregó el Tribunal que previo a ordenar el cumplimiento específico, se debe analizar si dicha medida de reparación no es desproporcionadamente mayor en cuanto a la soberanía del Estado que la indemnización monetaria. En palabras de Oscar SCHACHTER:

[S]i bien en principio corresponde cumplir la obligación de restablecer la situación que existía antes de la transgresión, puede haber razones materiales y sociales que hagan "imposible" o "impracticable" para el Estado ofensor el restablecimiento de la situación en su estado anterior ${ }^{55}$.

Por su parte, el Estado argumentó respecto dela supremacía de la soberanía estatal frente a las medidas no pecuniarias: "[A] un Estado soberano no puede ordenársele, contra su voluntad, restituir a un inversionista una inversión o concesión que se haya rescindido o expropiado. En esas circunstancias sólo puede exigírsele que pague una indemnización monetaria" ${ }^{\prime 56}$.

\footnotetext{
52. Ibídem.

53. Occidental v. Ecuador. N. 3.

54. Ídem, párr. 79.

55. Traducción libre. O. Schachter, International Law in Theory and in Practice, Collected Courses of the Hague Academy of International Law, 1982, p. 190.

56. Traducción libre. Ídem, p. 45.
} 
Sin perjuicio del criterio de este tribunal, cabe recalcar que ha pasado más de una década desde la expedición de esta decisión, por lo que los criterios respecto de las medidas reparatorias no económicas han evolucionado. Por ejemplo, en el caso Hela Schwarz GmbH v. República de China ${ }^{57}$, el tribunal arbitral explora la indiscutida aplicabilidad del cese y el cumplimiento específico como medidas para alcanzar la restitución integral.

\section{ConClusión}

En este artículo se exploró la naturaleza, aplicabilidad y ejecutabilidad de las medidas reparatorias no pecuniarias, así como los casos en los cuales el Ecuador ha sido parte. Después de un análisis doctrinario y jurisprudencial, se concluye que las medidas reparatorias no pecuniarias son completamente válidas, eficaces, exigibles, ejecutables y sobre todo compatibles contra la verdadera soberanía estatal. No obstante, los tribunales en más del $70 \%$ de los casos, ni siquiera consideran a este tipo de medidas como una opción válida para lograr reparar los daños perpetrados al inversionista. Particularmente, de la totalidad de los casos en los que el Ecuador ha participado, apenas en dos casos el tribunal ha concedido medidas de este tipo.

Es claro, que existe un latente e infundado temor por parte de los tribunales arbitrales internacionales en lesionar la soberanía estatal y por esta razón no consideran adoptar mecanismos distintos a las compensaciones monetarias. Al contrario, se ha zanjado la discusión sobre el verdadero alcance de la soberanía estatal, concluyendo así que un Estado no es menos soberano por cumplir decisiones ordenadas por tribunales arbitrales que contengan este tipo de medidas. Tal

57. Hela Schwarz GmbH v. República de China, Caso CIADI No. ARB/17/19. 
es así, que incluso se ha demostrado la posibilidad de llevar al arbitraje temáticas intrínsecamente ligadas a la soberanía del Estado como son disputas limítrofes.

Finalmente, el presente análisis no es una consigna para promover la adopción de medidas no pecuniarias como la única alternativa de reparación de daños en arbitraje internacional; toda vez que, se debe realizar un test de procedencia para determinar de manera objetiva si la aplicación de estas medidas protege un derecho existente y cumple con los parámetros de urgencia y necesidad que justifiquen su pertinencia. En vista de que, existen casos en los que las aplicaciones de estas medidas son de imposible cumplimiento o representan una carga desproporcionada respecto del beneficio que pudiera recibir el perjudicado. 